\title{
Extraction and characterization of Nanocellulose obtained from sugarcane bagasse as agro-waste
}

\author{
Mohammed B. Ghazy ${ }^{a^{\star}}$, Farag A. Esmail ${ }^{\mathrm{a}}$, Waleed K. El-Zawawy ${ }^{\mathrm{b}}$, Mariam. A. Al-Maadeed \\ and Medhat E. Owda ${ }^{a}$ \\ ${ }^{a}$ Chemistry Department, Faculty of Science, Al-Azhar University, Nasr City,11884 Cairo, Egypt. \\ ${ }^{\mathrm{b}}$ Cellulose and Paper Department, National Research Center, El-Tahrir St., Dokki, Cairo, Egypt \\ ${ }^{\mathrm{C}}$ Center for Advanced Materials, Qatar University, Doha, Qatar \\ Corresponding author E-Mail: medhatowda@gmail.com
}

ABSTRACT

This study aimed to characterize nanocellulose extracted from sugarcane bagasse (SCB) by acid hydrolysis $60 \%$ (w/w) $\mathrm{H}_{2} \mathrm{SO}_{4}$ at $45^{\circ} \mathrm{C}$. The effect of hydrolysis time $(20,30$ and $40 \mathrm{~min})$ on the structure and properties of the nanofibers was investigated. Fourier transform infrared spectroscopy (FT-IR), and X-ray diffraction (XRD) results indicated that the hemicellulose and lignin were removed extensively in the cellulose whiskers. The morphology and dimensions of the fibers and acid-released cellulose nanowhisker (CNW) were characterized by scanning electron microscopy (SEM) and transmission electron microscopy (TEM). The results showed that SCB could be used as source to obtain cellulose whiskers and they had needle-like structures. Longer hydrolysis time produced a lower yield of nanofibers; whereas the degree of crystallinity increased from $38.22 \%$ to $65.37 \%$ with increasing hydrolysis time due to removal of amorphous cellulose.

\section{Keywords}

Sugarcane bagasse- Cellulose whiskers- Acid hydrolysis- Nanocellulose

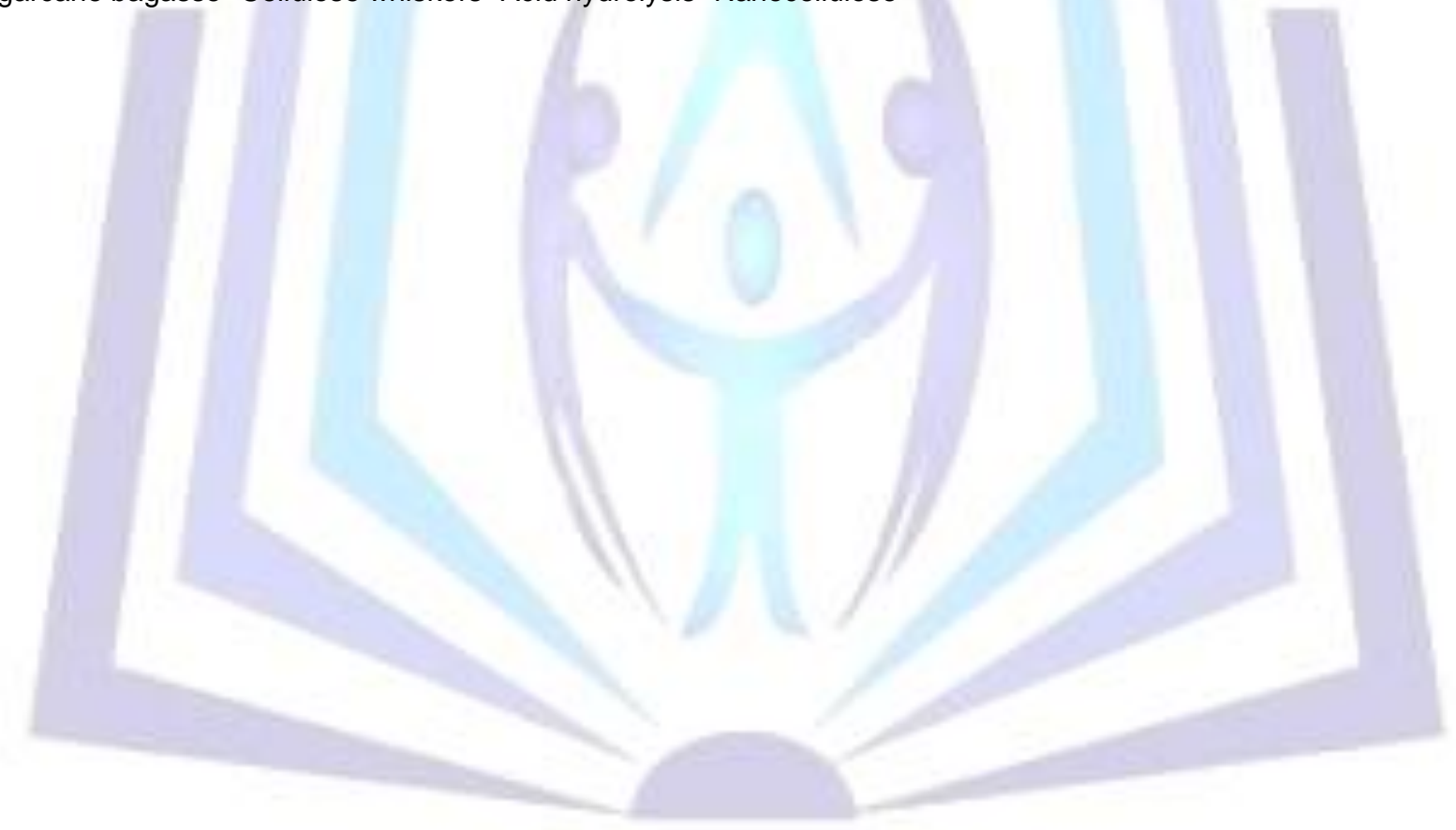

\section{Council for Innovative Research}

Peer Review Research Publishing System

\section{Journal: Journal of Advances in Chemistry}

Vol. 12, No. 3

editor@cirjac.com

www.cirjac.com 


\section{INTRODUCTION}

In recent years, the quest for sustainable development has motivated efforts toward maximizing the efficiency of the use of raw materials and minimizing the creation of waste (1). In this context, the use of biomass residues as feedstock for the production of energy and materials has been the object of intensive academic and industrial research (2-5). The reuse of these residues allows a significant reduction both in the volume of waste accumulated in the environment and in the extraction of raw materials. Thus, an efficient reuse of these wastes is of great importance, not only for minimizing the environmental impact, but also for obtaining a higher profit. Sugarcane bagasse (SCB) is a residue from the refining process of sugarcane that contains about $40-50 \%$ of cellulose in its composition (6). This characteristic suggests the possibility of using the SCB as a source of cellulose fibers for the extraction of whiskers structures. It is known that the morphology and properties of the CNW depend mainly on the source of the original cellulose, of the extraction process and their parameters (7-8). Therefore, the isolation and further analysis of the characteristics of CNW from many kinds of cellulosic resources is necessary and relevant for the efficient comparison and exploitation of these resources (9-11). CNW have been isolated from different vegetable sources, such as cotton and wood pulp $(7,12)$ and from animal sources such as tunicates (13). In addition, there are only a few papers which describe the isolation of whiskers from agricultural byproducts, such as soy hulls (10), corncob (11), rice husk (14) and sesame husk (15). Several processes (16-20) were used to extract cellulose nanowhiskers from biomass. They were generally based on successive cellulose fibers preparation and acid hydrolysis. Chemical treatments for cellulose fibers production at different routes were used to isolate nanowhiskers from mulberry (16), banana fibers (17), pea hull fibers (18), and sisal fibers (19-20).

The aim of this work was to isolate and characterize cellulose whiskers from bleached sugarcane bagasse treated with $(17.5 \%)$ sodium hydroxide $(\mathrm{NaOH})$ solution to extract cellulose followed by acid hydrolysis at a mild temperature $(45 \circ \mathrm{C})$, different time intervals $(20,30$ and $40 \mathrm{~min})$ and to investigate also the influence of extraction time on the morphology and crystallinity of the resulting whiskers.

\section{EXPERIMENTAL}

\subsection{Materials}

Bleached bagasse pulp was used for preparation of nanowhisker. It was kindly supplied by Qena Company for pulp and paper, Qena, Egypt. The pulp had the following composition: $26.8 \%$ hemicelluloses, $70.6 \%$ alpha cellulose, $0.82 \%$ ash content, $0.2 \%$ lignin. Sodium hydroxide $(\mathrm{NaOH})$, acetic acid, sodium bicarbonate and sulfuric acid reagent grade chemicals were purchased from Sigma-Aldrich.

\subsection{Isolation of Cellulose}

For alkali pretreatment, bleached bagasse pulp was treated with $17.5 \%$ sodium hydroxide for $1 \mathrm{hr}$ at room temperature to remove hemicellulose. At the end of extraction the insoluble residue (cellulose) was collected by filtration, washed thoroughly with water, dilute acetic acid ( $2 \%$ solution), with water again until neutral $\mathrm{pH}$ and finally air-dried.

\subsection{Preparation of Cellulose Nanocrystals}

The delignified and hemicellulose free cellulose was acid hydrolyzed at $45^{\circ} \mathrm{C}$ for $20 \mathrm{~min}, 30 \mathrm{~min}$ and 40 min with constant stirring. For each gram of cellulose $15 \mathrm{~mL}$ of $60 \% \mathrm{H}_{2} \mathrm{SO}_{4}(\mathrm{w} / \mathrm{w})$ was used. Immediately following the hydrolysis, the suspension was diluted 10-fold with distilled water to quench the hydrolysis reaction, and centrifuged twice for 10 min at $5000 \mathrm{rpm}$ to remove the excess acid then washed with water, $5 \%$ sodium bicarbonate and finally with distillated water to $\mathrm{pH}$ about 6 . Then, separating from the liquid phase at each washing by centrifugation. To obtain the dry nano-powder, the water dispersion of nanoparticles was sedimented, washed with acetone which can be separated from the solvent by centrifugation, and dried at $500 \mathrm{C}$ to constant weight. The cellulose nanocrystal samples were labeled SCBW20, SCBW30 and SCBW40 depending on the time of extraction. The mechanism of acid hydrolysis can be shown in figure (1)

\section{Characterization And Measurements}

\section{Yield Analysis}

The weight of the initial cellulose was designed as M1, while the SCBW was dried at 50 oC up to constant weight M2. The yield of SCBW is given as follows:

Yield \% $=\left(\mathrm{M}_{2} / \mathrm{M}_{1}\right) \times 100 \%$

\section{Fourier Transform Infrared Spectroscopy}

FT-IR spectroscopy (6100, JASCO model, 50 scans, Japan) was used to examine the changes in the functional groups induced by the various treatments. The samples were ground and mixed with $\mathrm{KBr}$ at $(1: 100)$ by weight, respectively. The resultant powder was pressed into transparent pellets and analyzed in absorbance mode within the range of $4000-400 \mathrm{~cm}$ 
(a)

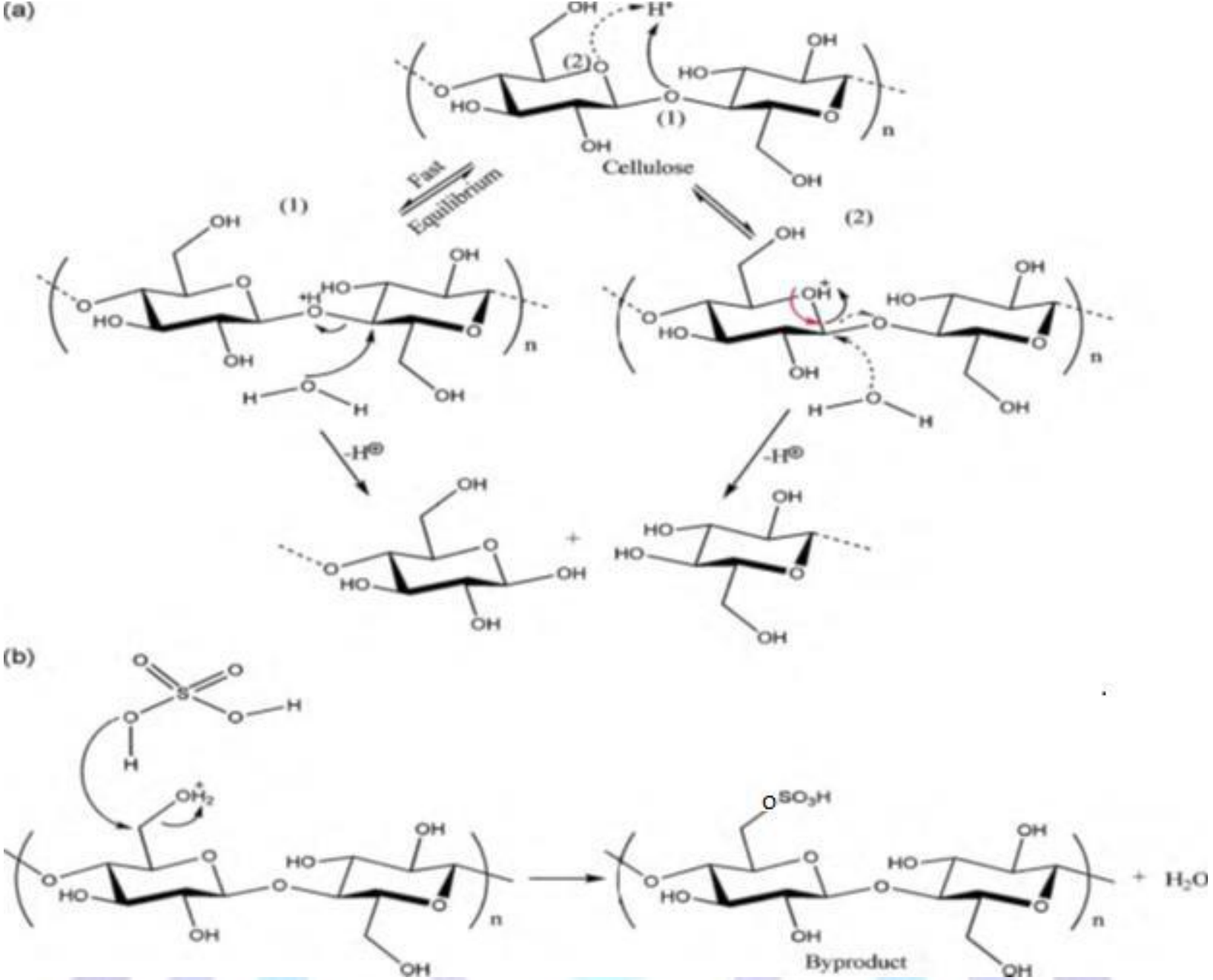

Fig (1): (a) Acid hydrolysis mechanism and (b) esterification of cellulose nanocrystals surfaces.

\section{Microscopic Analysis}

Morphological analysis of untreated, bleached and treated SCB was done by Scanning electron microscopy (SEM). SEM micrographs of fiber surface were taken using (JOEL JXA-840A electron probe microanalyzer with tungsten filament 30 Kv, Japan).

\section{Transmission Electron Microscopy (TEM)}

Transmission electron microscopy (TEM) is the one of most important imaging tools of nanotechnology in which a thin specimen is imaged by an electron beam which is irradiated through the sample at uniform current density. The size and shape of cellulose nanocrystals were investigated by transmission electron microscopy (JEOL JEM-2100 Electron Microscope, Japan). After the suspension was mildly ultrasonicated in water bath for $20 \mathrm{~min}$, A few drops of solution were dropped onto a TEM carbon coated grid and the residue was removed by a filter paper under the TEM grid.

\section{X-ray Diffraction (XRD) Analysis}

X-ray diffraction was used to determine the crystallinity of the SCB fibres after different treatments. Each sample in the form of milled powder was placed on the sample holder and levelled to obtain total and uniform X-ray exposure. The samples were analyzed using X-ray diffractometer at room temperature (RT) with a monochromatic Cu Ka radiation source $(\lambda=0.1539 \mathrm{~nm})$ in the step-scan mode with a $2 \Theta$ angle ranging from $5^{\circ}$ to $80^{\circ}$. To characterize the crystallinity of the different samples, the crystallinity index Ic, was determined based on the reflected intensity data following the equation of Segal et al. (21):

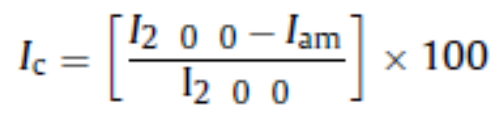

Where 1002 is the maximum intensity of the (002) lattice diffraction peak and lam is the intensity scattered by the amorphous part of the sample. The diffraction peak for plane (002) is located at a diffraction angle around $2 \theta=22 \circ$ and the intensity scattered by the amorphous part is measured as the lowest intensity at a diffraction angle around $2 \theta=18$. 


\section{Results and discussion}

\subsection{Nanocellulose Yields}

The yields of the SCBW, with respect to the initial amount of dried fibers, for SCBW20, SCBW30 and SCBW40 were $79.26,74$ and 65 wt\% respectively; these values are consistent with the literature data $(11,12,21)$. Among the several methods for preparing CNW, acid hydrolysis is the most well-known and widely used (22).This process breaks down the disordered and amorphous parts of the cellulose, releasing single and well-defined crystals. Thus, this process is based on the quicker hydrolysis kinetics presented by amorphous regions, as compared to crystalline ones (21-23). The decreasing yields with longer reaction time is expected as cellulose chains in the less ordered regions are hydrolyzed to separate the crystalline domains that reduce in sizes as the surface chains are further hydrolyzed, while cellulose fragments are broken into soluble oligo- and monosaccharides (24).

\subsection{FT-IR Spectroscopy}

Direct information about changes in chemical functionality can be obtained by FT-IR spectroscopy which has been extensively used for structural analysis of the material before and after chemical treatments. FT-IR spectra of SCB as raw material, chemically purified cellulose (CPC) and the SCBWs obtained by acid-hydrolysis are shown in Figure 2 . The spectral bands at $3344-3490$ and $2850-2970 \mathrm{~cm}^{-1}$ are characterized for the O-H stretching intramolecular hydrogen bonds (25) of hydroxyl groups and C-H stretching, respectively and the band at $1730 \mathrm{~cm}^{-1}$ represents the C-O stretching vibration for the acetyl and aster linkages in lignin, hemicellulose, pectin, etc. while the peaks at 1620-1649, 1512 and $1595 \mathrm{~cm}^{-1}$ are associated with the aromatic ring present in lignin and absorbed water, respectively and the peak at $1250 \mathrm{~cm}^{-1}$ corresponds to $\mathrm{C}-\mathrm{O}$ out of plane stretching due to the aryl group in lignin, which are associated with the SCB before the chemical treatments, are not observed for CPC. The effect of these chemical purification can be observed through main spectral bands specially the peak at $1735 \mathrm{~cm}^{-1}$, which is assigned mainly to $\mathrm{C}=\mathrm{O}$ stretching vibration of the carbonyl and acetyl groups in the xylan component of hemicellulose and also typical for structural features of lignin (26-27) disappeared after bleaching and alkaline treatments. Further absorption bands of lignin at approximately $1595 \mathrm{~cm}^{-1}(28)$ and, in particular, the band at $1512 \mathrm{~cm}^{-1}$ is absent (29-33). However, FT-IR spectra of SCBWs having sharp bands but similar to that observed in CPC. The spectral bands observed in the spectra of CPC and SCBWs in the region $1649-1634 \mathrm{~cm}^{-1}$ are assigned to the $\mathrm{O}-\mathrm{H}$ bending due to the adsorbed water (31). The peaks at $1430-1420,1382-1375 \mathrm{~cm}^{-1}$ and $893-902 \mathrm{~cm}^{-1}$ are allocated to the $\mathrm{CH} 2$ scissoring motion in cellulose, $\mathrm{C}-\mathrm{H}$ bending and $\mathrm{C}-\mathrm{O}-\mathrm{C}$ pyranose ring stretching vibration, respectively. While the two characteristics absorbtion bands at $893-902 \mathrm{~cm}^{-1}$ and $1155 \mathrm{~cm}^{-1}$ assigned to cellulosic $\beta$ glycosidic linkages and C-C ring stretching (33-34), respectively. The spectral bands observed at $1427 \mathrm{~cm}^{-1}$ and $897 \mathrm{~cm}^{-1}$ represent significant cellulose I content. Moreover, the bands at 1431, 1372, 1322, 1162, 1059 and $896 \mathrm{~cm}^{-1}$ are typical of pure cellulose (26-27)

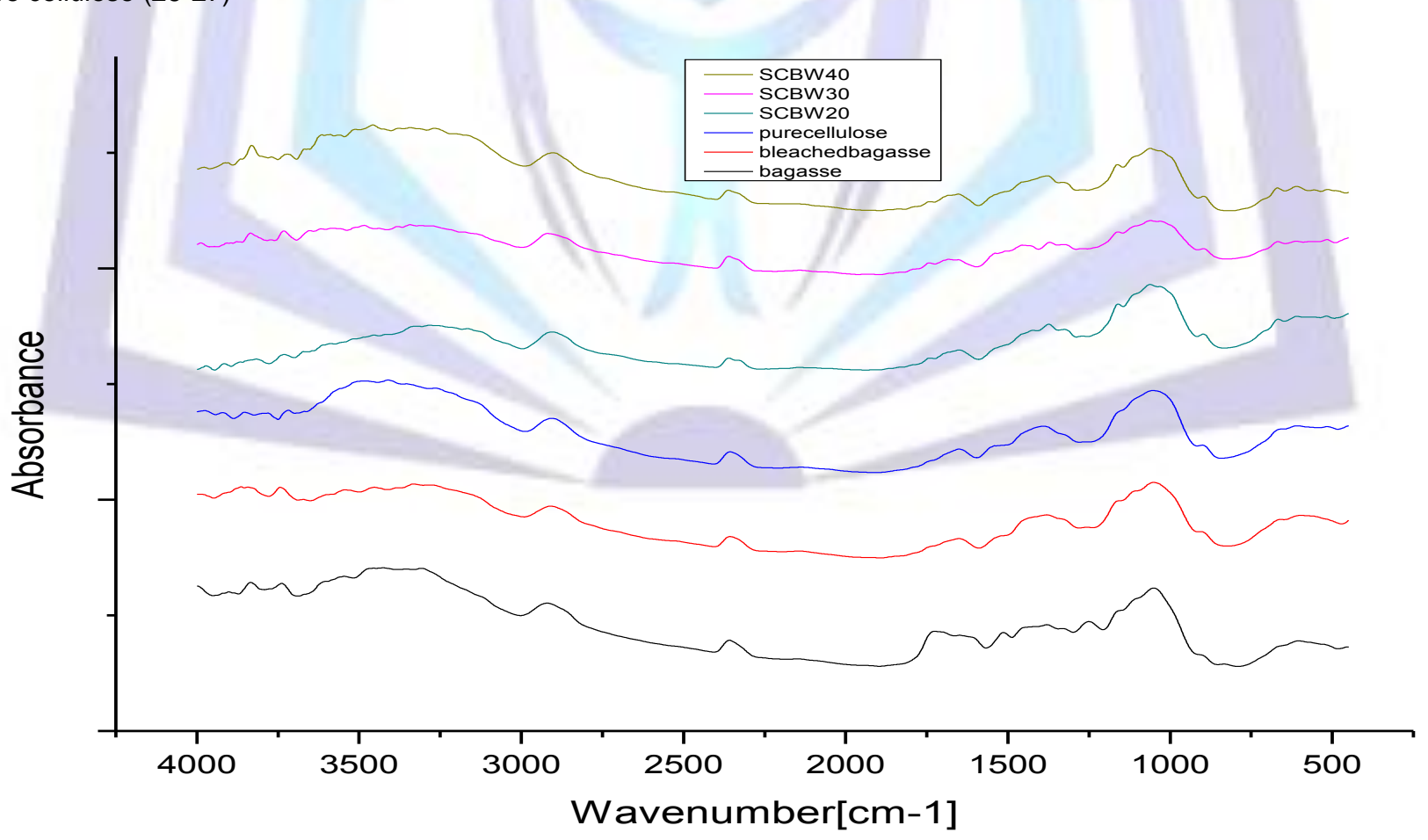

Fig (2): Infrared spectra of (a) bagasse (SCB) (b) bleached fibers (c) cellulose fib (d) SCBW20 (e) SCBW30 and (f) SCBW40.

\subsection{Scanning Electron Microscopy (SEM) Analysis}


The SEM micrographs of the original ground sugarcane bagasse (a), bleached bagasse pulp (b), pure cellulose after removal of lignin and hemicellulose (c) and nanocellulose produced byacid hydrolysis of the pure cellulose with $60 \%$ (w/w) $\mathrm{H}_{2} \mathrm{SO}_{4}$ at different time intervals $\left(20,30\right.$ and $40 \mathrm{~min}$.) while the temperature was kept constant at $45^{\circ} \mathrm{C}(\mathrm{d}, \mathrm{e}, \mathrm{f})$.
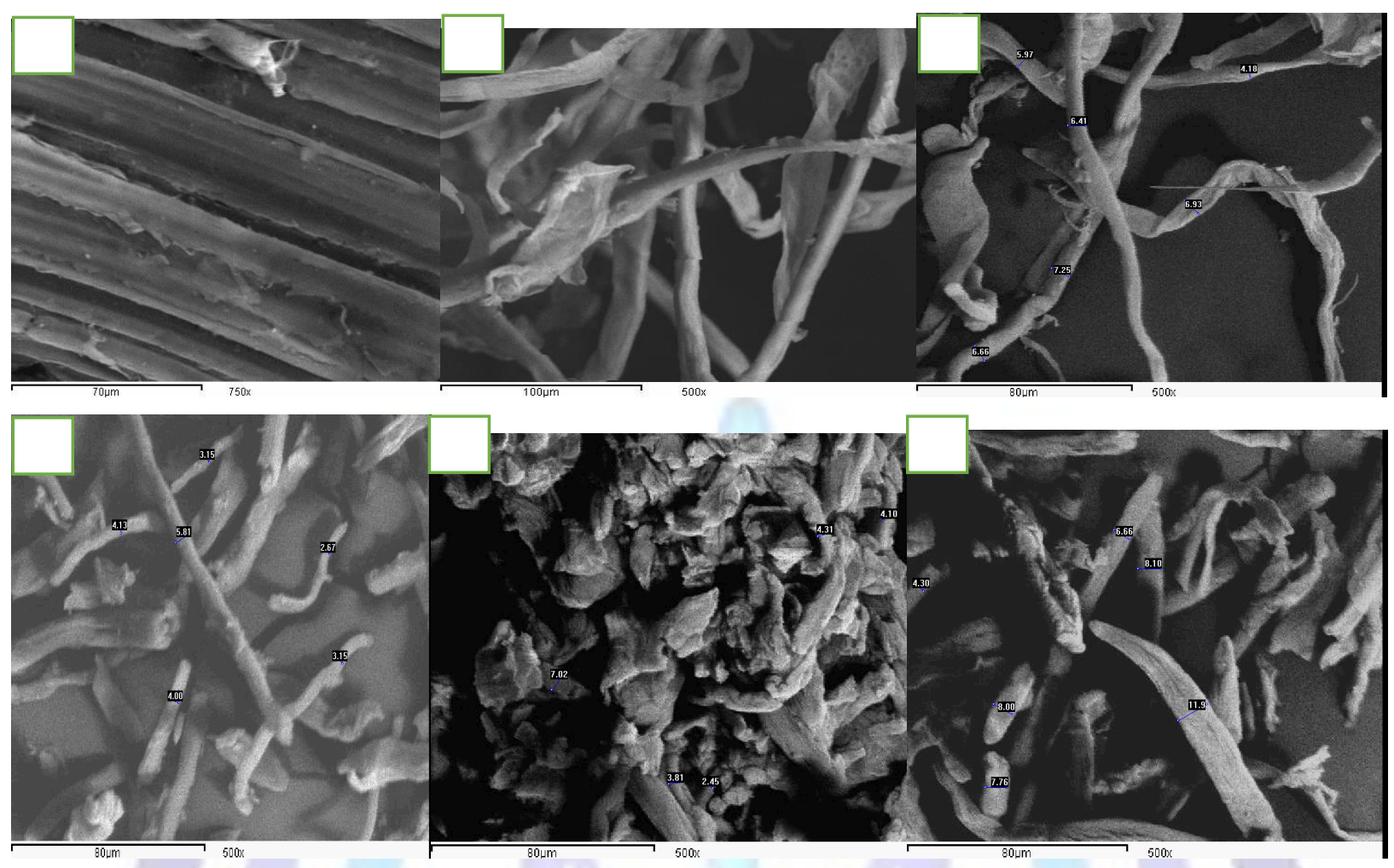

Fig 3. Scanning electron microscope of (a) bagasse (SCB) (b) bleached fibers (c) cellulose fibers (d) SCBW20 (e) SCBW30 and (f) SCBW40.

The diameter of the original sugarcane bagasse fiber was much bigger and each fiber appears to be composed of several microfibrils. Each elementary fiber possesses a compact structure; exhibiting an alignment in the fiber axis direction. In its natural state, and before chemical extraction, the overall surface of the fiber is found to be somewhat smooth due to the presence of waxes and oil (35-36) and other incrusting materials such as hemicellulose, lignin and pectin that form a thin layer to protect the cellulose inside. On treating with bleaching agent the lignin is removed through complex formation and depolymerization. On subsequent treatment with alkali the hemicellulose is hydrolyzed and becomes water soluble. These help in defibrillation of the fibrils and result in micrograph (b) and (c) whereby the diameter of the fibrils is reduced to agreat extent, also possibly because of removal of non-cellulosic constituents. Untreated microcrystalline cellulose (MCC) displays irregular aggregated shape fibrils and a rough surface morphology with lower aspect ratio, these aggregates composed of strong hydrogen bonding between hundreds of individual cellulose whiskers (37-39). In the cellulose fiber, the sulfuric acid hydrolysis usually could cleavage the amorphous regions of microfibrils transversely, resulting in a diameter reduction of fibers from micron to nanometer (40) as shown in Table (1). Hydrolyzing of cellulose from tunicates and green algae produced whisker like particles with a length of micrometer, where higher plants cellulose from cotton and wood pulp gave shorter particles a few hundred of nanometers in length (8). The hydrolyzed fibers at different times have a rod-like shape. After acid hydrolysis of SCB at different time intrvals, it's clear from typical SEM micrograph that the aggregation of MCC was broken down and the tendency of fiber separation can clearly be observed after these treatments gave rise to intermittent fibrillar structure and further reduction in intrafibrillar diameter especially after 30 minutes.

Table (1): Diameter $(\mu \mathrm{m})$ determination by image j program from SEM micrograph.

\begin{tabular}{|c|c|}
\hline Sample & Diameter $(\boldsymbol{\mu m})$ \\
\hline Bagasse & 392.15 \\
\hline Bleached pulp & 16.02 \\
\hline Pure cellulose & 9.531 \\
\hline SCBW 20 & 4.748 \\
\hline SCBW 30 & 4.708 \\
\hline SCBW 40 & 5.28 \\
\hline
\end{tabular}




\subsection{Transmission Electron Microscopy (TEM)}

In order to determine fiber length, and to indicate the aggregation state of the whiskers, highly diluted samples of the hydrolyzed suspension were analyzed by transmission electron microscopy. At least 10 measurements for each condition were used to determine average and standard deviation values.

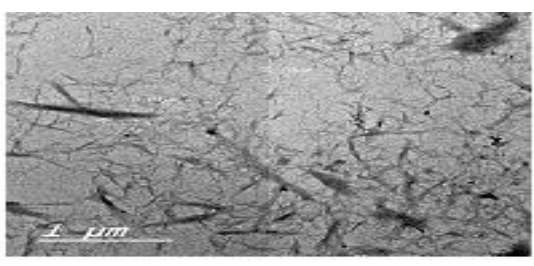

a

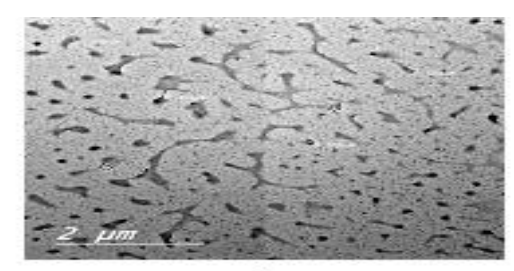

b

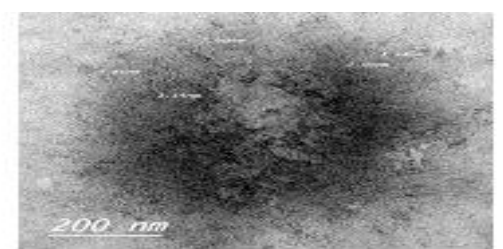

C

Fig 4. TEM image of cellulose nanowhiskers (a) SCBW20 (b) SCBW30 (c) SCBW40

These images evidenced the efficiency of the acid hydrolysis treatment, confirming that the bagasse cellulose nanowhiskers consisting mostly of individual fibrils and some aggregates. (40-41). The effect of preparation conditions on the dimensions of cellulose nanowhiskers were characterized by particle length (L), width (D) and aspect ratio (L/D). As pointed out by Dong et al. (42), the conditions of preparation govern somewhat the properties of the cellulose particles. It is expected that the prolonged acid attack can not only "dissolve" amorphous molecules but also partly destroy crystalline zones of the fibrils, ultimately resulting in a shortening of nanowhisker length. However, the results show that, for the tested conditions, the values are differences between the lengths of nanowhiskers obtained from the different conditions of preparation. Typically, bagasse nanowhiskers had lengths ranging from 7 to $80 \mathrm{~nm}$ and width from 1.7 to $40 \mathrm{~nm}$.

\subsection{X-ray Diffraction (XRD) Analysis}

Cellulose has crystalline structure contrary to hemicellulose and lignin, which are amorphous in nature. According to Kumar et al. (29) pointed out that cellulose has a crystalline structure due to hydrogen bonding interactions and Van der Waals forces between adjacent molecules. X-ray diffraction (XRD) analysis was completed to evaluate the crystallinity of the SCB fibres after different chemical treatment stages. Chemical treatment performed on natural fibres can affect the crystallinity of cellulose. For example, dilute acid has no effect on the crystalline domains, but destroys the amorphous region of the fibre (43). On the other hand, the alkali treatment can be performed onto the plant fibres to increase the stiffness of fibre as the impurities present in the fibres can be removed during this treatment (44). Therefore, the crystallinity of chemically treated fibres can be determined and compared to untreated fibres to access the effectiveness of the chemical treatment. Fig. (5) Shows the diffraction patterns obtained for (a) untreated, (b) alkali treated, (c) bleached, and (d-f) acid hydrolysed SCB. The crystallinity index was determined for the various samples and the results are summarized in Table (2). A continuous increase of the Crl value was observed upon the successive chemical treatments. The highest value $(65.37 \%)$ corresponds to cellulose nanocrystals, which also displayed the strongest and sharpest peak at $2 \theta=22^{\circ}$. The increased crystallinity following SCB treatments compared to the raw material was ascribed to the progressive removal of amorphous non-cellulosic materials. The subsequent increase of the $\mathrm{Crl}$ value upon acid hydrolysis of purified cellulose SCB fibres is indicative of the dissolution of amorphous cellulosic domains. During the hydrolysis process, hydronium ions can penetrate the more accessible amorphous regions of cellulose and allow the hydrolytic cleavage of glycosidic bonds, which eventually releases individual crystallites (45). In addition, during the preparation of cellulose nanocrystals the growth and realignment of monocrystals may occur in parallel and thus can improve the cellulose crystallinity (16). This phenomenon could explain the narrowing of the diffraction peaks. This increase in the cellulose fibres crystallinity was also expected to increase their stiffness and rigidity, and therefore strength. Thus, it was assumed that the potential mechanical properties and reinforcing capability of treated fibres increased (46-47).

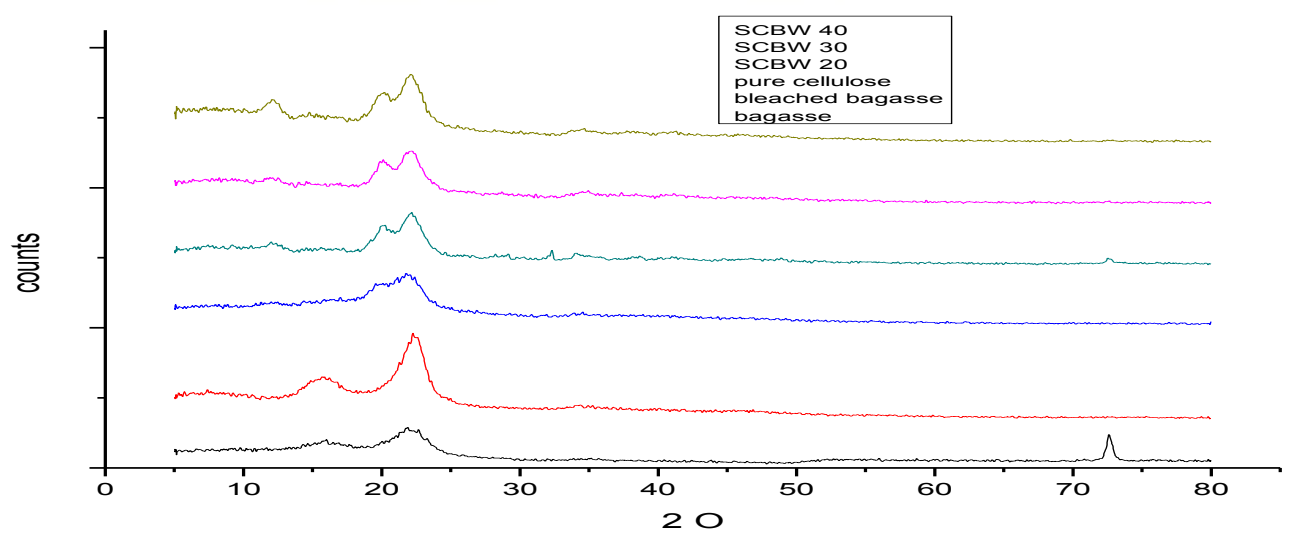

Fig 5. XRD of (a) bagasse (SCB) (b) bleached fibers (c) cellulose fib (d) SCBW20 (e) SCBW30 and (f) SCBW40. 
Table (2): Crystallinity index (IC) of SCB fibres at different stages of treatment.

\section{CONCLUSION}

\begin{tabular}{|c|c|}
\hline Sample & $\mathbf{I}_{\mathbf{c}} \%$ \\
\hline untreated SCB & 38.22 \\
\hline Pure cellulose & 48.41 \\
\hline Bleached SCB & 53.44 \\
\hline SCBW20 & 54.03 \\
\hline SCBW30 & 57.99 \\
\hline SCBW40 & 65.37 \\
\hline
\end{tabular}

Cellulose fibers were obtained from SCB fibers by alkali and bleaching treatment which are characterized by chemical analysis, FT-IR spectroscopy, and microscopic observations. Cellulose nanocrystals were successfully extracted from purified material using sulfuric acid while keeping the acid concentration and reaction temperature constant and varying the hydrolysis time. The chemical structure of the nanoparticles did not change during the acid hydrolysis process. Individually released cellulose nanocrystals showed an increased crystallinity when the reaction time was increased to 40 min. SCB whiskers were obtained at mild temperature $\left(45^{\circ} \mathrm{C}\right)$ and shorter extraction times $(20,30$ and 40 min). Their dimensions can be accurately measured by TEM and exhibited good crystallinity (65.37\%) by XRD measurements.

\section{REFERENCES}

[1] Ashori, A., "Wood-plastic composites as promising green-composites for automotive industries," Bioresource Technology, 99, (2008), 4661-4667.

[2] Mishra, S., Mohanty, A. K., Drzal, L.T., Misra, M., and G. Hinrichsen, "A review on pineapple leaf fibres, sisal fibres and their biocomposites," Macromolecular Materials and Engineering, 289, (2004), 955-974.

[3] Reddy, N.,Yang, Y., "Structure and properties of high quality natural cellulose fiber from cornstalks," Polymer 46(15), (2005), 5494-5500.

[4] Schievano, A., D'Imporzano,G., and Adani,F., "Substituting energy crops with organic wastes and agroindustrial residues for biogas production," Journal of Environmental Management, 90, (2009), 2537-2541.

[5] Moraisa, J. P. S., Rosab, M. F., Filhob, M. M. S., Nascimentoa, L. D, Nascimentob, D,M., and Cassalesb,A,R., "Extraction and characterization of nanocellulose structures from raw cotton linter," Carbohydrate Polymers, 91, (1) 2, (2013), 229-235, 2013.

[6] Sun, X. F., Sun, R. C., Su,Y and Sun, J. X., "Comparative study of crude and purified cellulose from wheat straw," Journal of Agricultural and Food Chemistry, 52, (2004), 839-847.

[7] Beck-Candanedo, S., Roman, M., and Gray, D. G., "Effect of reaction conditions on the properties and behavior of wood cellulose nanocrystal suspensions", Biomacromolecules, 6, (2005), 1048-1054.

[8] Elazzouzi-Hafraoui, S., Nishiyama, Y., Putaux, J., Heux, L., Dubreuil, F., and Rochas, C., "The shape and size distribution of crystalline nanoparticles prepared by acid hydrolysis of native cellulose," Biomacromolecules, 9, (2008), 57-65.

[9] Chen, W.S., Yu, H. P., Liu, Y. X., Chen, P., Zhang, M. X., and Hai, Y. F., "Individualization of cellulose nanofibres from wood using high-intensity ultrasonication combined with chemical pretreatments," Carbohydrate Polymer, 83, (2011), 1804-1811.

[10] Flauzino, W. P., Neto, H.A. Silvério, N.O. Dantas, and D. Pasquini, "Extraction and characterization of cellulose nanocrystals from agro-industrial residue - soy hulls," Industrial Crops and Products 42, (2013), 480-488.

[11] Silvérioa, H. A., Neto, W. P. F., Dantas, N. O., and Pasquini, D., "Extraction and characterization of cellulose nanocrystals from corncob for application as reinforcing agent in nanocomposites," Industrial Crops and Products, 44, (2013), 427-436.

[12] Teixeira, E. M., Correa, A.C., Manzoli, A., Leite, F. L., Oliveira, C. R., and Mattoso, L. H. C., " Cellulose nanofibers from white and naturally colored cotton fibers," Cellulose, 17, (2010), 595-606.

[13] Berg, O., Capadona, J. R., and Weder, C., "Preparation of homogeneous dispersions of tunicate cellulose whiskers in organic solvents," Biomacromolecules 8, (2007),1353-1357.

[14] Rosa, S. M. L., Rehman, N., De Miranda, M. I. G., Nachtigall, S. M. B., and Bica, C. I. D., "Chlorine-free extraction of cellulose from rice husk and whisker isolation," Carbohydrate Polymers, 87, (2012), 11311138. 
[15] Purkait, B. S., Ray, D., Sengupta, S., Kar, T., Mohanty, A., and Misra, M., "Isolation of cellulose nanoparticles from sesame husk. Industrial Engineering Chemistry Research 50, (2011),871-876.

[16] Li, R., Fei, J., Cai, Y., Li, Y., Feng, J., and Yao, J. " Cellulose whiskers extracted from mulberry: A novel biomass production," Carbohydrate Polymers, 76, (2009), 94-99.

[17] Cherian, B. M., Pothan, L. A., Chung, T. N., Mennig, G., Kottaisamy, M., and Thomas, S., "A novel method for the synthesis of cellulose nanofibril whiskers from banana fibres and characterization," Journal of Agriculture and Food Chemistry, 56, (2008), 5617-5627.

[18] Chen, Y., Liu, C., Chang, P. R., Cao, X., and Anderson, D. P., "Bionanocomposites based on pea starch and cellulose nanowhiskers hydrolyzed from pea hull fibre: Effect of hydrolysis time," Carbohydrate Polymers, 76, (2007), 607-615.

[19] Morán, J. J., Alvarez, V. A., Cyras, V. P., and Vázquez, A., "Extraction of cellulose and preparation of nanocellulose from sisal fibers," Cellulose, 15, (2008), 149-159.

[20] Segal, L.C., Martin, A. E., and Conrad, C. M., "An empirical method for estimating the degree of crystallinity of native cellulose using X-ray diffractometer," Textile Research Journal, 29, pp.786-794, 1959.

[21] Teixeira, E.de M., Bondancia, T. J., Teodoro, K. B. R., Correa, A. C., Marconcini, J. M., and Mattoso, L. H. C., "Sugarcane bagasse whiskers: Extraction and characterizations," Industerial Crops and Products, 33, (2011), 63-66.

[22] Peng, B. L., Dhar, N., Liu, H. L., and Tam, K. C., "Chemistry and applications of nanocrystalline cellulose and its derivatives: a nanotechnology perspective," Canadian Journal of Chemical Engineering, 89, (2011),1191-1206.

[23] Habibi, Y., Lucia, L. A., and Rojas, O. J., "Cellulose Nanocrystals: Chemistry, Self-Assembly, and Applications," Chemical Reviews, 110, (2010),3479-3500.

[24] Jiang, F., Hsieh, Y. L., "Chemically and mechanically isolated nanocellulose and their self-assembled structures," Carbohydrate Polymers, 95, (2013), 32- 40.

[25] Pan, M., Zhou, X., and Chen, M., "cellulose nanowhisker isolation and properties from acid hydrolysis combined with high pressure homogenization," Bioresources, 8(1), (2013),933-943.

[26] Ibrahim, M. M., El-Zawawy, W. K., and Nassar, M. A., "Synthesis and characterization of polyvinyl alcohol I nanospherical cellulose particle films," Carbohydrate Polymers, 79, (2010), 694-699.

[27] Ibrahim, M. M., Dufresne, A., El-Zawawy, W. K., and Agblevor, F, A., "Banana fibers and microfibrils as lignocellulosic reinforcements in polymer composites," Carbohydrate Polymers, 81, (2010), 811-819.

[28] Agblevor, F. A., Ibrahim, M. M., and El-Zawawy, W. K., "Coupled acid and enzyme mediated production of microcrystalline cellulose from corn cob and cotton gin waste," Cellulose, 14, (2007), 247-256.

[29] Kumar, A., Negi, Y. S., Bhardwaj, N.K., and Choudhary, V., "Synthesis and characterization of methylcellulose/PVA based porous composite," Carbohydrate Polymer, 88, pp. 1364-1372, 2012.

[30] Viera, R. G. P., Filho, G.R., de Assuncao, R. M. N., Meireles, C. S., Vieira, J. G., and de Oliveira, G .S., "Synthesis and characterization of methylcellulose from sugarcane bagasse cellulose," Carbohydrate Polymer, 67, (2007),182-189.

[31] Troedec, M., Sedan, D., Peyratout, C., Bonnet, J., Smith, A., Guinebretiere, R., Gloaguen, V., and Krausz, $P$., "Influence of various chemical treatments on the composition and structure of hemp fibers," Composites Part A- Applied Science and Manufacturing, 39, (2008), 514-522.

[32] Sain M., and Panthapulakkal, S., "Bioprocess preparation of wheat straw fibers and their characterization," Industerial Crops and Product, 23, (2006),1-8.

[33] Garside, P., and Wyeth, P., "Identification of cellulosic fibres by FTIR spectroscopy: Thread and single fibre analysis by attenuated total reflectance," Stud. Conser, 48, (2003),269-275.

[34] Mandal, A., and Chakrabarty, D., "Isolation of nanocellulose from waste sugarcane bagasse (SCB) and its characterization”, Carbohydrate Polymer, 86, (2011),1291-1299.

[35] Doraiswammy, I., and Chellamani, P., "Pineapple-leaf fibers," Text Programs, 24(1), (1993),1-37.

[36] Garcia, C., Jaldon, G., Dupeyre, D., and Vignon, M. R., "Fibres from semi retted hemp bundles by steam explosion treatment," Biomass Bioengineering, 14, (1998), 251-260.

[37] Liu, D., Zhong, T., Chang, P. R., Li, K., and Wu, Q., "Starch composites reinforced by bamboo cellulosic crystals," Bioresource Technology, 101, (2010), 2529-2536.

[38] Mathew, A. P., Oksman, K., and Sain, M., "Mechanical properties of biodegradable composites from poly lactic acid (PLA) and microcrystalline cellulose (MCC)," Journal of Applied Polymer Science, 97, (2005), 
2014-2025

[39] Petersson, L., Kvien, I., and Oksman, K., "Structure and thermal properties of poly (lactic acid)/cellulose whiskers nanocomposite materials," Composites Science and Technology, 67, (2007), 2535-2544.

[40] Azizi Samir, M. A. S., Alloin, F., and Dufresne, A., "Review of recent research into cellulosic whiskers, their properties and their application in nanocomposite field," Biomacromolecules, 6(2), (2005), 612-626.

[41] Lai-Kee-Him, H., Chanzy, H., Müler, M., Putaux, J. L., Imai, T., and Bulone, V., "In vitro versus in vivo cellulose microfibrils from plant primary wall synthases: Structural differences," The Journal of Biological Chemistry, 277(40), (2002),36931- 36939.

[42] Dong, X. M., Revol, J. F., and Gray, D. G., "Effect of microcrystallite preparation conditions on the formation of colloid crystals of cellulose," Cellulose, 5, (1998), 19-32.

[43] Fengel, D., and Wegener, G., "Wood: Chemistry, Ultrastructure, Reactions. Walter de Gruyter, Berlin, 1984.

[44] Mwaikambo, L. Y., and Ansell, M. P., "Chemical modification of hemp, sisal, jute, and kapok fibers by alkalization," Journal of Applied Polymer Science, 84, (2002), 2222-2234.

[45] de Souza Lima, M. M., Borsali, R., "Scattering and Optical Microscopy Experiments of Polyelectrolyte Rodlike Cellulose Whiskers," in: Concentrated Dispersions: Theory, Experiments, and Applications, ACS Symp. Ser., 878, Am. Chem. Soc., Washington, DC 2004

[46] Bhatnagar, A., and Sain, M., "Processing of cellulose nanofiber-reinforced composites," J. Reinforced Plastic and Composite. 24, (2005),1259-1268.

[47] Rong, J., Jing, Z., Li, H., and Sheng, M., "A Polyethylene Nanocomposite Prepared via In-Situ Polymerization," Macromolecule Rapid Communication, 22, (2001),329-334. 